Running head: METHODS IN PREFERENTIAL LOOKING

What am I supposed to be looking at? Controls and measures in inter-modal

\author{
preferential looking \\ Katie Alcock \\ Lancaster University \\ Sarah Watts \\ City University, London \\ Jessica Horst \\ University of Sussex
}

Address for correspondence:

Department of Psychology

Fylde College

Lancaster University

LA1 4YF

Tel: (+44) 01524593833

Fax: (+44) 01524593744

Email:k.j.alcock@lancaster.ac.uk 


\begin{abstract}
Intermodal preferential looking (IMPL) is widely used in experimental studies of infant development, especially language development. Control measures vary, and it is not clear how these affect findings. We examined effects of parental awareness of stimuli. Infants (17-19mo) looked at paired pictures, one name-known and one nameunknown, each assigned target status in $50 \%$ of trials. Infants looked longer at a name-known than a name-unknown target, regardless of parents' awareness. When parents were aware, looking to a name-unknown target increased over a paired nameknown non-target. There is evidence that infants' looking at pictures in this paradigm is not due to direct matching of targets to novel names, but is influenced by additional cues present, in a way that could alter the conclusions of studies of infant word learning and other aspects of infant learning. Implications of these findings are discussed, emphasising replicability and theoretical conclusions drawn from studies using this method.
\end{abstract}

Keywords:

Intermodal preferential looking; Language development; Experimental techniques; Nonverbal cues 


\section{What am I supposed to be looking at? Controls and measures in inter-modal preferential looking}

\section{Intermodal preferential looking}

The intermodal preferential looking (IMPL) technique is so termed because it involves presenting an infant with stimuli in two modalities - hearing a word or sound whilst a choice of visual stimuli - pictures (moving or still) or objects - is presented to the infant in different locations. The infant's looking times (in many studies, both latency of looking - the equivalent of reaction time - and time spent looking), to each stimulus is recorded and conclusions are drawn about infants' knowledge from the differences between or preferences for one stimulus over another. Use of this technique in language development research is described in detail in an early paper by Thomas, Campos, Shucard, Ramsay, and Shucard (1981) and has since been used extensively in language acquisition research as well as for examining non-linguistic development (Bebko, Weiss, Demark, \& Gomez, 2006).

In the context of language acquisition research, IMPL has been used to investigate development of the lexicon (Golinkoff, Hirsh-Pasek, Cauley, \& Gordon, 1987; Houston-Price, Plunkett, \& Harris, 2005; Naigles \& Gelman, 1995; Schafer, Plunkett, \& Harris, 1999), syntax (Naigles, 1998) and phonology (Werker, Fennell, Corcoran, \& Stager, 2002). It is also a useful technique for studying the skills of children with disabilities, who may not otherwise be able to respond (Cauley, Golinkoff, Hirsh-Pasek, \& Gordon, 1989; Walker-Andrews, Haviland, Huffman, \& Toci, 1994). Intermodal preferential looking (IMPL or alternative abbreviations) is the term of choice in most recent studies (Bailey \& Plunkett, 2002; Golinkoff, Ma, Song, \& Hirsh-Pasek, 2013; Houston-Price, Mather, \& Sakkalou, 2007; HoustonPrice et al., 2005; Tan \& Schafer, 2005; Tincoff \& Jusczyk, 1999) although older 
papers use different terms (Ballem \& Plunkett, 2005 among others: preferential looking; Forbes \& Poulin-Dubois, 1997 among others: visual preference) and no name was given to the paradigm in Thomas et al.’s (1981) paper. Fernald, Zangl, Portillo, and Marchman (2008) describe frame-by-frame analysis of infants' looking as "looking while listening", described by Golinkoff et al. (2013) as "the next generation" of preferential looking.

\section{Parent cuing in IMPL}

Early studies using IMPL emphasised the benefits of the technique for experimental control. Some authors stressed the need for control over the parent's awareness of the stimuli (Thomas et al., 1981). When children do not know the correct answer to a question they often look for additional cues from the adults around them (see Grow \& LeBlanc, 2013 for a review of possible inadvertent cues). Subtle differences from adults in terms of verbal emotional valence (e.g. Mumme \& Fernald, 2003), accent (van Heugten \& Johnson, 2014) and tiny pauses before target words (e.g. Read, Macauley, \& Furay, 2014) significantly influence where children look and point in experiments. Infants are also sensitive to non-verbal cues and gestures such as eye-gaze (e.g Grossmann \& Johnson, 2010), emotional facial expressions (e.g. Repacholi, 1998) and previous reliability (e.g. Tummeltshammer, Wu, \& Kirkham, 2013). If parents were to provide infants with non-verbal cues - either intentionally to help them succeed in the task, or inadvertently-it is likely that infants would respond to these cues. Controlling for parent awareness reduces parents' ability to bias the data by providing cues.

There are many ways to control for parent awareness and cuing when using the IMPL technique. For example, in the experimental setup used by Thomas et al. (1981) "the mother sat behind and faced away from her infant and the displays and 
therefore did not know where the target objects were located nor how her baby was responding” (p.800). Naigles (1990) gave mothers a visor to wear during testing. Likewise in Golinkoff et al.'s (1987) study mothers were asked to close their eyes during testing (though it is not reported whether all complied). Swingley and Aslin (2000) asked parents to direct their gaze downwards and excluded data from an infant whose parent peeked (see also Swingley, 2009). Other labs have used dark glasses with tape on the inside (e.g. Fernald et al., 2008).

However, in some studies parents have been allowed to view the stimuli presented - and in many more studies to listen to them. For example, Reznick and Goldfield (1992) positioned parents where "they could not influence the child's gaze." Parents "could see the slides but were discouraged from participating in any way" (p.408). Infants were in a high chair, rather than on parents' laps, it is not stated whether parents could or did touch their infants during testing. Delle Luche, Durrant, Poltrock, and Floccia (2015) only asked parents not to interfere. Halberda (2003) allowed parents to see and hear the stimuli, but to "maintain forward gaze and not cue the infants verbally or physically" (p. B25) whilst infants were seated on parents' laps. Four infants had to be excluded from the study, because their parents violated these instructions. Golinkoff (1987) and Swingley (2009; Swingley \& Aslin, 2000) have a mechanism for preventing parents from seeing the stimuli but they can still hear.

In many further studies parents have been allowed to hear the stimuli (Candan et al., 2012; Dittmar, Abbot-Smith, Lieven, \& Tomasello, 2008; Fernald et al., 2008; Forbes \& Poulin-Dubois, 1997; Naigles, 1990; Naigles \& Tovar, 2012). Parents are therefore aware of the words children are hearing during the experiment. In some labs, the setup varies depending on the participant group. For example, in Candan et 
al. (2012) parents of Turkish and English learning children were told to close their eyes but it appears that parents of Mandarin learning children could see the visual stimuli. For children with autism, Naigles and Tovar (2012) recommend a setup where parents do not hear the stimuli, but can see them. The same lab uses a setup where the parents can hear, but not see, for other participant groups (Naigles \& Gelman, 1995). However, whether parental awareness is worth controlling for has not been systematically explored. It remains unclear whether parents can intentionally or inadvertently influence infants' looking behaviour when the parents are able to see and hear the stimuli.

\section{The current study}

This study will investigate the impact of parental awareness on IMPL. It will compare infants' looking to pictures associated with known and unknown words when parents can see and hear the stimuli, versus when parents cannot see or hear the stimuli. We hypothesised that when parents can see and hear the stimuli infants' looking will be influenced, probably in the direction of increased looking at the targets.

This disambiguation task was chosen not as a direct test of the mutual exclusivity assumption (Markman \& Wachtel, 1988; Mervis \& Bertrand, 1994), but so we could compare conclusions drawn from studies using similar methods with and without parental controls. To facilitate comparison to other studies using IMPL we compared looking time to an unknown target to the level expected by chance $(50 \%$ of the available looking time).

In addition, for a subset of parents we coded the variety of behaviours that parents engage in when they are able to see and hear the stimuli. We expect these 
behaviours to occur whether those targets have names that are known or unknown to the infant, as it is assumed all targets are known to the parents. The most likely cues from parents are expected to be primarily looking at the name-unknown target, but also speaking and pointing (Grow \& LeBlanc, 2013). We hypothesised that infants would be more likely to look at the unknown targets on trials in which parents used such cues. Note, the current study is not designed to investigate theoretical issues in language acquisition, but rather to examine the difference that experimental controls used in data collection make to the conclusions that are drawn from studies using IMPL. Finally, we will also compare reaction times to determine whether parent awareness can affect reaction times.

\section{Method}

\section{Participants}

Infants were recruited through invitation letters circulated at local nurseries in the south-east of England. In total 22 infants aged 17 to 19 months participated, with a mean age of 18.3 months $(S D=1.00)$. Infants were excluded if they had a history of hearing loss or developmental delay, or if either parent or any caregiver spoke a language other than English to them. Infants were brought to the lab by their mother in all cases. No infants were excluded for other reasons.

\section{Stimuli}

\section{Choice of stimuli.}

On arrival at the lab, parents completed a 74-item checklist of words (object names) likely to be known by infants of this age, chosen to be a) imageable and b) likely to be known by more than $15 \%$ of 16-month-olds (Dale \& Fenson, 1996). Parents indicated whether their child knew each word on the list (either in comprehension or production). For each infant 8 to 10 words that were known and 8 
to 10 that were not known were chosen, attempting to balance the number of times each word was used as a known and an unknown stimulus across infants. The stimulus pool and the number of times each word was used as a known or an unknown stimulus is shown in Appendix 1. For a few words (e.g. aeroplane, train, onion) it became clear part way through testing that all infants either knew or did not know the word, so balancing was not possible for some words. Stimuli used were hence different for each infant, based on their word knowledge as reported by parents.

\section{Visual stimuli.}

The experimenter also checked with the parent that each infant would be familiar with a physical or depicted exemplar of each of the words, whether or not they knew the word. Hence visual stimuli were all of familiar objects. Visual stimuli were adapted from clipart taken from a CD-ROM of common infants' vocabulary (Dorling Kindersley, 2000), with additional items acquired from a variety of websites. Visual stimuli consisted of a colour picture of each item on a white background, 300 by 300 pixels, the picture taking up $75 \%$ to $90 \%$ of the white area in at least one dimension.

\section{Auditory stimuli.}

The sentence "Look! Look at the X" was digitally recorded in a sound-proof setting using an intonation patterned on naturalistic UK English child-directed speech for each stimulus and was played to the child starting simultaneously with the onset of the two pictures. Sound files had a mean length of $2300 \mathrm{msec}$ (range 2100 to 2600).

\section{Setup.}

Visual stimuli were projected using a digital projector onto a screen facing the infant, who was seated on their mother's lap. The projected pictures (white background with photograph of the stimulus) were approximately $30 \mathrm{~cm}$ square, on a 
black background, with a $30 \mathrm{~cm}$ space between the two pictures, and the infant and mother were seated on a chair approximately $1 \mathrm{~m}$ from the screen. Auditory stimuli were presented via a central pair of speakers located behind the projection screen and a central digital video camera located between the two pictures recorded infants' faces and mothers' faces and upper bodies (mothers for participants 13 through 22 only).

\section{Procedure}

Pictures were presented in pairs, one of which was a name-known picture and one a name-unknown picture. Infants were presented with two blocks of 20 pairs of pictures. Pictures were presented for 6 seconds followed by an inter-trial interval of 6 seconds of a plain black screen. Each known-name picture was always paired with the same unknown-name picture for a given child and the child heard the names for both pictures. Order and side were counterbalanced across trials. Thus, the child encountered each picture in four configurations across the two blocks: Left picture is known, known name is heard; right picture is known, known name is heard; left picture is known, unknown name is heard; right picture is known, unknown name is heard. The known-name picture appeared once as the target and once as the nontarget during each of the two blocks.

During one block the mother wore a sleep mask and headphones, over which she could hear classical music (the "Unaware" condition). During the other block, mothers could see and hear the stimuli (the "Aware" condition); the order of blocks was counterbalanced across mothers. There was a short break between the two blocks during which infants and mothers could move around the laboratory or play, to maintain infants' attention during the second block, and to enable mothers to put on or take off the headphones and sleep mask. Mothers reported that they could not hear or see the stimuli during the Unaware block. 
Mothers were not given details of the purpose of the experimental manipulation before testing but were told to try not to "tell their child what to do" or to "help their child," regardless of the condition. Mothers who enquired about the purpose of the manipulation were asked to wait until after testing, at which point they were debriefed. Parent behaviour was videoed in addition to infant looking for half of the infants. Unfortunately no video of parents was available for the other parents.

Testing started when infants were attending to the screen, and could be paused between trials if infants looked away. An attention getter sound (chimes) and display (red circles on a black background) were used if infants looked away between trials and did not return their own gaze to the visual display.

\section{Analysis Strategy}

After testing infants' looking was coded frame-by-frame by one of three observers (Author 1, Author 2 or a student volunteer), who could see which two pictures infants viewed during each trial but who could not hear the sounds that infants heard, nor did they know which of the pictures was name-known and which was name-unknown for each particular infant. For half of the infants, parent behaviour was also coded (see below for details). Onset and offset of looks to each picture were noted. For each trial the following were coded:

- total time spent looking at each picture before the auditory stimulus ended (baseline looking time), examined in ANOVA 1

- Total time spent looking at each picture after the auditory stimulus ended ANOVA 2a; proportion of all looking to each picture after the auditory stimulus, ANOVAs $2 \mathrm{~b}$ and $2 \mathrm{c}$, which respectively analyse all time after the sound ends and time during the "looking window" suggested by Fernald et al. (2008). Added in to this is an analysis of looking time to targets as a 
proportion of all post-sound looking time, compared to chance $(50 \%)$ This is the only comparison that can determine whether children are looking more to the target picture than the non-target, without the potential confound of absolute looking time to target plus to distractor being limited by the time for which the picture is presented.

- reaction time of the first look to each picture over the whole trial (baseline RT), examined in ANOVA 3.

- reaction time of the first look to each picture after the auditory stimulus ended, and during the "looking window", examined in ANOVAs 4a and 4b.

Many previous studies categorise baseline looking as looking before the auditory stimulus starts (Houston-Price et al., 2007; Schafer et al., 1999). Although it might be possible for infants to identify the referent of the auditory stimulus before its offset, it would not be possible to code the disambiguation point for each individual infant (e.g. an infant who knew "cat" but not "camera" might have an earlier disambiguation point than one who knew both words), so for one of our analyses the end of each sound file was taken as the point at which to code post-stimulus looking.

Note, this also takes into account Swingley \& Aslin (2000)'s concerns about minimum possible reaction times to a visual stimulus. Where a look started before the offset of the sound file but continued after its offset, the infant was still counted as looking at the picture after the sound file, but only the portion of the look after the sound file was counted. The Fernald et al. (2008) "looking window" also takes into account these concerns, but using a somewhat arbitrary start point, that cannot determine when a child can disambiguate a word.

While Fernald et al. (2008) suggest only counting looks where the child was, 
before the auditory stimulus was presented, looking at the distractor stimulus, this seems a false exclusion to us. If a child is looking away, or looking at the target, and hears the instruction "Look! Look at the X!" then the correct action is to begin looking at the target (if looking away) or to continue looking at the target (if already looking at it). Hence, we counted all looking times to the target after the "looking window" started, or after the sound file ended, whatever the child's behaviour was before the measurement start point.

Mothers' behaviour during the Aware block was coded in the same way by an observer (Author 2) who was unaware of both the auditory stimulus and which picture was name-known. The observer coded whether the mother spoke, pointed, moved her head, or looked and in which direction. Although sound was not available for the mothers' videos, it was possible to lip-read some infants repeating the stimulus noun, but no mother could be seen to do this.

\section{Results}

For each of the dependent variables, initially a $2 \times 2 \times 2$ within participants ANOVA was carried out which compared the effects of parent awareness, known word status (whether parents indicated infants knew a word for the object) and Target status of each picture (whether it was named during the trial or not). Order and side were collapsed within analyses.

\section{Results of ANOVAs}

Baseline time spent looking: before offset of auditory stimulus - ANOVA

1.

Here the total looking time was compared between parent awareness conditions as a test of the hypothesis that parents awareness may increase overall 
looking time in general. Total looking time to both stimuli rarely if ever approaches the total time for which stimuli are presented so that infants' total looking time to each stimulus is also interesting - without analysing this we cannot determine if attention is increased when parents are aware of the stimuli. There was no significant effect of parent condition, known/unknown word status or target/non-target picture status (see Table 1). There were also no significant interactions. This makes intuitive sense because in this phase parents did not yet know what the target picture was regardless of condition.

[Table 1 about here]

\section{Total time spent looking after offset of auditory stimulus - ANOVA 2a.}

For a check on the independence of target vs non-target looking time, a correlation was carried out between the total looking time after the offset of the sound to all targets vs all non-targets. There was no correlation between time spent looking to the target and time spent looking to the non-target, $r(22)=.15, p=.50$. That is, the time spent looking to the target did not reduce the time spent looking to the nontarget.

First, we compared the total looking time from the offset of auditory stimulus to offset of visual stimulus to test the hypothesis that parent awareness could affect total looking time. A significant 3-way interaction was found between parent awareness condition, known word status, and target picture status, $F(1,21)=5.30, p$ $=.032, \eta^{2}=.20$. In the Unaware condition, infants looked longer at name-known targets than at name-unknown targets. However, in the Aware condition, infants looked for comparable times at both name-known and name-unknown targets (see below for post-hoc tests). This interaction can be seen in Figure 1. Thus, parents' 
awareness of the target influenced the likelihood that infants would look at nameunknown pictures in response to words they did not yet know.

[Figure 1 about here]

There were no significant 2-way interactions; between condition and target picture status, $F(1,21)=3.00, p=.10$, between condition and known word status, $F(1,21)=0.48, p=.50$, and between target picture status and known word status, $F(1,21)=1.33, p=.26$. There were significant main effects of target picture status, $F(1,21)=35.10, p<.001, \eta^{2}=.63$, and of known word status, $F(1,21)=17.56, p$ $<.001, \eta^{2}=.46$, indicating that infants looked longer to the correct pictures and to the pictures for known-name objects in general. There was again no main effect of parent awareness condition, $F(1,21)=.05, p=.83$. This indicates that across stimuli infants are just as likely to engage with and look at the stimuli when the parent is wearing a blindfold and headphones than when the parent is allowed to see and hear. In addition, we find no evidence that infants are distracted when parents are blindfolded or wear headphones.

Post-hoc analyses examined the difference between target and non-target looking in four conditions: Unaware with the target name-known, Unaware with the target name-unknown, Aware with the target name-known and Aware with the target name-unknown. These confirmed the significant three-way interaction found above. Where the target was name-known, looking was significantly longer to the target than the non-target in both Unaware and Aware conditions, $t(21)=6.26, p<.001, d=1.73$ for Unaware, $t(21)=5.16, p<.001, d=1.89$ for Aware. For name-unknown targets looking was longer to the target in the Aware condition but this was not significant, $t(21)=1.80, p=.086, d=.63$, but for name-unknown targets in the Unaware 
condition there was little difference between looking at the target and the non-target $t(21)=.04, p=.97, d=0.01$.

ANOVAs examining proportion of looking time - note that these ANOVAs are two-way.

Proportion of total looking time spent looking to target after offset of sound - ANOVA 2 b.

A significant main effect of known word status was found, $F(1,21)=16.73, p$ $=.001, \eta^{2}=.44$. The effect of parent awareness condition was not significant, $F$ $(1,21)=2.05, p=.17, \eta^{2}=.09$, and the interaction between word status and parent condition was not significant either, $F(1,21)=.61, p=.44, \eta^{2}=.03$.

Proportion of total looking time during "looking window" spent looking at target - ANOVA 2c

A significant main effect of whether the target was known was again found, $F(1,21)=14.04, p=.001, \eta^{2}=.40$. The effect of parent awareness condition was not significant $F(1,21)=2.08, p=.74, \eta^{2}=.09$, and the interaction between condition and known status was not significant either.

\section{Proportion of looking time to target compared to chance.}

Infants' proportion of looking time to the target was compared to chance (0.50) for each combination of parent awareness and known word status (that is the four conditions: Unaware known-name target, Unaware unknown-name target, Aware known-name target and Aware unknown-name target), using the Holm-Bonferroni correction. When the target was name-known looking to the target was significantly greater than would be expected by chance both when the parent was aware $(M=.63$, $S D=.10 t(21)=4.93, \mathrm{p}<.001)$ and when the parent was unaware $(M=.63, S D=.13$ $t(21)=6.06, p<.001)$. This is what we would expect if infants really do know the 
words. As such, this analysis demonstrates that parents were accurate in their reporting of which words infants' knew. However, when parents were aware and infants did not know the names of the objects their looking times were marginally longer than expected by chance $(M=.56 S D=.13 t(21)=2.22, p=.037$, n.s. with correction for multiple comparisons). Critically, when parents were unaware of the stimuli infants did not look to the name-unknown target more than chance $(M=.50$, $S D=.07, t(21)=.14, p=.89)$. That is, infants looked to targets more than half the time only when they knew the names. This analysis of proportions replicates the findings of ANOVA 2 a for raw looking times.

\section{Baseline reaction time: reaction time of first look in trial - ANOVA 3.}

No significant main effects of parent awareness condition, Known word status or Target picture status or interactions were found (see Table 1), indicating that parent awareness itself did not affect looking time

\section{RT of first look after offset of sound - ANOVA 4.}

Reaction time of first look was coded as the first look that started or continued after the offset of the auditory stimulus. If a look started before the offset of the sound but continued after it, the reaction time was coded as zero. An ANOVA revealed no significant main effects of Known status, $F(1.21)=.246, \mathrm{p}=.63, \eta^{2}=.01$, Target status $F(1,21)=.00, p=.98, \eta^{2}=.00$, or parent condition $F(1,21)=1.63, p=.216$, $\eta^{2}=.07$, nor any significant interactions (see Table 1 ).

\section{RT of first look to target during "looking window" - ANOVA 4b}

Fernald et al. (2008) suggest only coding reaction time for looks to targets only, that start during the looking time window rather than those that continue during this window. Using this measure, there was no significant effect of whether the target was known $F(1,21)=.095, p=.76, \eta^{2}=.01$, nor of condition $F(1,21)=.372, p=.55$, 
$\eta^{2}=.03$, and no significant interaction.

\section{Parent interaction effects}

For half of the parents, parent bodies were filmed and parent behaviour was coded. All parents were observed to either point, look, move their head or speak at some point during the Aware block. The most common behaviour was speaking (13\% of trials, $60 \%$ of parents) followed by looking to a name-unknown non-target ( $11 \%$ of trials, $20 \%$ of parents). Parents looked both to targets and non-targets ( $7 \%$ of trials versus $4.5 \%$ of trials), and both to name-known and name-unknown pictures ( $4 \%$ of trials versus $7.5 \%$ of trials). An ANOVA examining parent looking behaviour to targets/non-targets and known/unknown name pictures, revealed no main effects of Target status or Known status, and there was no interaction. However, only half of these parents looked to one side or the other (the rest of parents, and some of these parents, pointed or spoke).

Although parents varied widely in the type of potential cues they made, all parents performed some potential cue behaviour on some trials. An ANCOVA was carried out, covarying the proportion of trials on which each parent performed any potentially cuing behaviour at all to examine the effect of body movements on infant looking time (after offset of sound). A significant main effect of target was found, $\left.F(1,8)=7.40, p=.026, \eta^{2}=.48\right)$, but no main effects of awareness condition, or of known word status were found, $F(1,8)=.29, p=.61, \eta^{2}=.03, F(1,8)=.64, p=.45, \eta^{2}$ $=.07$ respectively, nor any significant interactions between independent variables or the covariate parent behaviour. It seems that although parent awareness condition may be able to explain some aspects of looking behaviour, it is not clear that all differences in infant looking between the Aware and Unaware conditions can be explained by the presence or absence of overt parent cuing behaviour. 
Taken together, the results from the analysis of parents' behavioural cues, ANOVA 2a and the comparison of infants' proportion of looking time against chance, it is clear that when parents are aware of the stimuli infants respond differently toward name-unknown object pictures than when parents are unaware. This has direct consequences for how we evaluate research findings from IMPL tasks.

\section{Discussion}

\section{Parent awareness and parent cueing}

The current study explored the effect of parents' awareness of stimuli on infants' looking times during an IMPL experiment. We found that patterns of infants looking were different when parents were aware of auditory and visual stimuli. Overall, we found no quantitative differences in total looking time, but qualitative differences in which stimulus infants looked at longest. Specifically, we found a significant interaction between looking to a known vs unknown target, and parent awareness, meaning that infants were looking longer at name-unknown targets than the corresponding name-known non-targets, but only when parents could see and hear. This may be due to parents providing infants with non-verbal cues (e.g., looking, gesturing or shifting seating position) or due to infants' own awareness that parents could see and hear leading to an increase in their own interest or an attempt to engage parents' interest. These possibilities are not mutually exclusive: both may be contributing to the effect.

Our finding of no overall change in looking time is crucial given the widespread assumption among researchers that children will be distracted and fail to pay attention to stimuli if their parent is blindfold or wearing headphones. In our study, infants did not appear to be distracted, and crucially total looking time over all 
conditions was the same whether or not parents could see and hear the stimuli. Researchers do not explicitly outline this assumption in their academic articles but they also do not justify allowing parents to see and/or hear stimuli in any other way (Golinkoff et al., 1987; Halberda, 2003; Houston-Price et al., 2007; Reznick \& Goldfield, 1992; Swingley, 2009; Swingley \& Aslin, 2000); the procedures are simply presented without any explanation.

The possibility of increased infant attention if parents are not blindfolded or wearing headphones is however frequently expressed informally, and some experimenters explicitly suggest that specific groups need more and different adjustments (Candan et al., 2012; Naigles \& Tovar, 2012). It is vital that similar studies are carried out with these special groups before such an assumption can be made. Another possible control might be pre-training the parent and child so they are familiar with the setting before introducing blindfolds and headphones.

In the current study, parent awareness did not simply influence infants to look more overall, nor did it influence looking at target/non-target, or name-known/nameunknown pictures equally. Rather, allowing parents to see and hear stimuli altered infants' looking in a way that would lead researchers to draw different conclusions from the same experimental paradigm. The significant interaction we found suggests that when parents could see and hear the stimuli infants behaved as if they were applying a linguistic principle like mutual exclusivity (Markman, 1990); N3C, (Mervis \& Bertrand, 1994); disjunctive syllogism, (Halberda, 2003). When parents could not see and hear the stimuli infants did not demonstrate this behaviour. Note that our study was not intended as a direct test of these linguistic principles, but rather an experiment to see what conclusions might be drawn in attempts to test such 
theories of language acquisition, where controls are either in place or not in place.

Some previous researchers have stressed experimental control, ensuring parents are unaware of both visual and auditory stimuli (Naigles, 1990; Thomas et al., 1981), but others have not, or have controlled parents' looking more informally, usually by asking parents to close their eyes, seating them where they cannot see, or just asking them not to influence the child's looking (Golinkoff et al., 1987; Halberda, 2003; Houston-Price et al., 2007; Reznick \& Goldfield, 1992; Swingley, 2009; Swingley \& Aslin, 2000). Our findings suggest that researchers should pay close attention to parents' ability to view the stimuli. Although the sample size for which we have a direct measurement of behaviour is small, when we control for parent behaviour (the analysis of co-variance carried out above) infants still look more to target than non-target pictures. However, once parent behaviour was controlled for, infants did not look longer at known-name than unknown-name pictures (despite our findings in earlier analyses that did not control for parent behaviour). It is unclear whether this analysis controlling for parent behaviour would replicate over the whole sample, though, or a larger sample, because of the lack of information about parent behaviour for the whole sample.

From these data, we cannot distinguish, though, between the effects of parent visual awareness and parent auditory awareness. There is more data available to date on awareness by infants of parent vision than of parent hearing. However, many of the studies that fail to control for parent awareness do not in fact control for either type of awareness.

Although many researchers have emphasised analysing proportional looking time (e.g. Fernald et al., 2008; Schafer et al., 1999) looking time differences (e.g. 
Golinkoff et al., 1987), and reaction time (Fernald et al., 2008), others have analysed raw looking time (Golinkoff et al., 1987; Naigles, 1990; Werker et al., 2002), and used raw looking time enabled us to draw conclusions about the potential influences of different conditions on infants' overall attention - an important consideration in IMPL studies. It may turn out that either blindfolding or masking noise is sufficient to control for parent awareness, and it is reassuring to know that even when both are done it does not reduce infant looking time.

As we predicted, it is difficult to draw conclusions about the mechanism of this effect of parent awareness. This could be a parent effect (parents cue infants when they are aware of the stimuli), a child effect (infants' attention is increased when they know parents are aware, or infants attempt to engage parents more in some conditions), or both. Likewise, it could be a conscious effect (e.g. infants actively try to find parents' cues which in the past have given them reliable information about stimuli) or a subconscious effect (e.g. infants feel parent movement in one direction and this causes them to move or look in that direction). In addition, it is not yet clear whether parents give infants cues intentionally or inadvertently. Alternatively, although overall looking time does not increase when parents are aware, it is possible that infants' distress or distraction when parents are not aware leads to additional task demands, which do not decrease overall looking time, but instead alter task performance.

\section{Choice of data for analysis}

Some previous studies have examined proportions of looking, collapsing target and non-target looking into the same variable. Indeed, it could be said that whatever statistic can possibly be calculated from IMPL data has been used by at least 
one infant laboratory. Here, we compared baseline and post-auditory stimulus looking times and pre- and post-auditory stimulus reaction times . In our analysis of postauditory-stimulus looking times and reaction times we chose two looking windows: the onset and offset times recommended by Fernald et al. (2008), from 300msec after the onset to $1800 \mathrm{msec}$ after the onset of the auditory stimulus, and an alternative window from the offset of the auditory stimulus to the end of the trial. Because Fernald and colleagues explain they chose that window on relatively arbitrary grounds, we felt it was important to also analyse a time window in which we could be completely certain that all children definitely had access to the identity of the auditory stimulus (i.e. after the auditory stimulus finished) and not to artificially terminate the analysis window early. Our approach enables us to examine differences that have not been seen in other studies, while still controlling for them.

Different statistics taken from the same trial may or may not inter-correlate, depending on a variety of factors including whether or not looking times to one picture or the other, or in different portions of the trial, are independent. We did not find a significant correlation between looking time to the target and non-target, suggesting that these are independent variables and therefore assumptions of statistical tests are met when comparing these. We ensured that we were truly carrying on analyses on independent values by going on to compare the proportion of looking time in each condition to chance, and the same results was obtained.

Also, our analyses find differences in some comparisons but not in others, in some cases, suggesting that studies using different outcome measures may come to different conclusions. It has proved helpful to compare a variety of measures in this study. For example, we found no target effects on looking time before the auditory 
stimulus was heard, meaning looking effects are not due to an inherent preference for the target picture but rather depend on hearing the auditory stimulus.

\section{Future Directions}

Our data challenge some of the assumptions and make important methodological recommendations for studies using IMPL. We would urge cautious interpretation of studies that allowed parents to see and/or hear stimuli. When we did not specifically tell parents not to look at the stimuli, we observed several types of overt parent behaviours, which may have potentially cued infants. Since blindfolding parents and providing masking auditory stimuli did not cause infants to decrease their overall looking times, it would seem advisable for blindfolds and auditory masking to become standard in infant testing setups.. Our masking stimulus - music - also needs further investigation. While clearly our blindfold and auditory masking made a difference in some conditions, it is possible that masking was not wholly effective, especially in the auditory dimension. For example, Pinto, Fernald, and Mc Roberts (1998) report that even edited music tracks (with no pauses) are not fully effective as masks for auditory stimuli.

In addition, future research should examine more closely the parent behaviours that cue infants and the results of strict attempts to control parent behaviour. Instructing parents to keep still, look at their infant rather than at the stimuli, and not to speak, may or may not affect infants' behaviour in the same way: if infants are being directly cued by parent behaviour, then when parents are aware of the stimuli but reduce their cues, infant behaviour may change. If on the other hand, infants are changing their behaviour because of their awareness that parents can see visual stimuli, asking parents to remain motionless and not look at stimuli, but not 
blindfolding them, may still affect results. Generally, researchers exclude children where parents have obviously interfered with a trial (Naigles \& Tovar, 2012), but

parents' behaviour may not need to be obvious for children to perform differently. Whenever studies show theoretically "mature" behaviour (as with infants' looking at name-unknown targets) it would be helpful to know what the impact of parent cues, or of infants' awareness of parents' awareness, might be on this behaviour. Is this effect a general one that leads to behaviour that might be classified as more "mature", or does parental awareness only affect behaviour in certain circumstances?

To our knowledge, there are no data to confirm whether infants of this age are aware of others' ability to hear auditory stimuli; this would also be helpful to investigate. Some studies (Swingley, 2009) restrict parents' viewing of stimuli without auditory interference. However, parents can judge in-the-moment whether their child knows a word or not, and again could potentially react in a way that influences children's behaviour. This is particularly the case when pseudowords are used.

\section{Conclusions}

We have made an important contribution both to the literature on the IMPL paradigm but also to the body of knowledge on infant testing in general. Critically, parent awareness influences infant looking behaviour, which can bias results in IMPL tasks and affect how we evaluate these results and the theories they support. Labs vary widely in their use of parent controls and also in their elimination of trials and infants based on the types of behaviours we have observed here. Tighter control of parent awareness does not alter infants' participation rate, indexed by overall looking time, but could avoid elimination of infants and trials in a way that can lead to study bias. Given the influence parent awareness can have on infant looking behaviour in 
IMPL tasks, our study is timely and our findings have broader implications. 


\section{Author note}

We would like to acknowledge the assistance of members of the info-childes mailing list who enthusiastically responded to a plea for information on methodology in their labs' studies. In particular, Letitia Naigles responded extremely kindly by sending us details of her lab's whole body of work in this area with notations on the methodology used.

Portions of the data from this study formed part of the BSc dissertation of Author 2 . 


\section{References}

Bailey, T. M., \& Plunkett, K. (2002). Phonological specificity in early words. Cognitive Development, 17(2), 1265-1282.

Ballem, K. D., \& Plunkett, K. (2005). Phonological specificity in children at 1;2. Journal of Child Language, 32(1), 159-173.

Bebko, J. M., Weiss, J. A., Demark, J. L., \& Gomez, P. (2006). Discrimination of temporal synchrony in intermodal events by children with autism and children with developmental disabilities without autism. Journal of Child Psychology and Psychiatry, 47(1), 88.

Candan, A., Küntay, A. C., Yeh, Y.-c., Cheung, H., Wagner, L., \& Naigles, L. (2012). Language and age effects in children's processing of word order. Cognitive Development, 27(3), 205-221.

Cauley, K. M., Golinkoff, R. M., Hirsh-Pasek, K., \& Gordon, L. (1989). Revealing hidden competencies: A new method for studying language comprehension in children with motor impairments. American Journal on Mental Retardation, 94(1), 53.

Dale, P., \& Fenson, L. (1996). Lexical development norms for young children. Behavior Research Methods, Instruments and Computers, 28, 125-127.

Delle Luche, C., Durrant, S., Poltrock, S., \& Floccia, C. (2015). A methodological investigation of the Intermodal Preferential Looking paradigm: Methods of analyses, picture selection and data rejection criteria. Infant Behavior and Development, 40, 151-172. doi:https://doi.org/10.1016/j.infbeh.2015.05.005

Dittmar, M., Abbot-Smith, K., Lieven, E., \& Tomasello, M. (2008). Young German children's early syntactic competence: A preferential looking study. Developmental Science, 11(4), 575-582. 
Dorling Kindersley. (2000). DK Originals Children's Dictionary [CD-ROM]. London: Dorling Kindersley.

Fernald, A., Zangl, R., Portillo, A. L., \& Marchman, V. A. (2008). Looking while listening: Using eye movements to monitor spoken language. Developmental psycholinguistics: On-line methods in children's language processing, 44, 97.

Forbes, J. N., \& Poulin-Dubois, D. (1997). Representational change in young children's understanding of familiar verb meaning. Journal of Child Language, 24(2), $389-406$.

Golinkoff, R. M., Hirsh-Pasek, K., Cauley, K. M., \& Gordon, L. (1987). The eyes have it: lexical and syntactic comprehension in a new paradigm. Journal of Child Language, 14(1), 23-45.

Golinkoff, R. M., Ma, W. Y., Song, L. L., \& Hirsh-Pasek, K. (2013). TwentyFive Years Using the Intermodal Preferential Looking Paradigm to Study Language Acquisition: What Have We Learned? Perspectives on Psychological Science, 8(3), 316-339. doi:10.1177/1745691613484936

Grossmann, T., \& Johnson, M. H. (2010). Selective prefrontal cortex responses to joint attention in early infancy. Biology Letters, 6(4), 540-543.

Grow, L., \& LeBlanc, L. (2013). Teaching Receptive Language Skills. Behavior analysis in practice, 6(1), 56-75.

Halberda, J. (2003). The development of a word-learning strategy. Cognition, 87(1), B23-B34.

Houston-Price, C., Mather, E., \& Sakkalou, E. (2007). Discrepancy between parental reports of infants' receptive vocabulary and infants' behaviour in a preferential looking task. Journal of Child Language, 34, 701-724.

Houston-Price, C., Plunkett, K., \& Harris, P. (2005). 'Word-learning wizardry' 
at 1;6. Journal of Child Language, 32(1), 175-189.

Markman, E. M. (1990). Constraints Children Place on Word Meanings. Cognitive Science, 14(1), 57-77.

Markman, E. M., \& Wachtel, G. F. (1988). Children's use of mutual exclusivity to constrain the meaning of words. Cognitive Psychology, 20(2), 121.

Mervis, C. B., \& Bertrand, J. (1994). Acquisition of the Novel NameNameless Category (N3C) principle. Child Development, 65(6), 1646-1662.

Mumme, D. L., \& Fernald, A. (2003). The infant as onlooker: Learning from emotional reactions observed in a television scenario. Child Development, 74(1), 221237.

Naigles, L. (1990). Children use syntax to learn verb meanings. Journal of Child Language, 17(2), 357-374.

Naigles, L. (1998). Developmental Changes in the Use of Structure in Verb Learning: Evidence From Preferential Looking. Advances in Infancy Research, 12, 298-318.

Naigles, L., \& Gelman, S. A. (1995). Overextensions in comprehension and production revisited: Preferential-looking in a study of dog, cat, and cow. Journal of Child Language, 22(1), 19.

Naigles, L., \& Tovar, A. T. (2012). Portable intermodal preferential looking (IPL): Investigating language comprehension in typically developing toddlers and young children with autism. Journal of visualized experiments: JoVE(70).

Pinto, J., Fernald, A., \& Mc Roberts, G. (1998). Reliability and validity in infant auditory preference procedures. Advances in Infancy Research, 12, 278-297.

Read, K., Macauley, M., \& Furay, E. (2014). The Seuss boost: Rhyme helps children retain words from shared storybook reading. First Language, 34(4), 354-371. 
Repacholi, B. M. (1998). Infants' use of attentional cues to identify the referent of another person's emotional expression. Developmental Psychology, 34(5), 1017.

Reznick, J. S., \& Goldfield, B. A. (1992). Rapid change in lexical development in comprehension and production. Developmental Psychology, 28(3), 406-413.

Schafer, G., Plunkett, K., \& Harris, P. L. (1999). What's in a name? Lexical knowledge drives infants' visual preferences in the absence of referential input. Developmental Science, 2(2), 187.

Swingley, D. (2009). Onsets and codas in 1.5-year-olds' word recognition. Journal of Memory and Language, 60(2), 252-269.

Swingley, D., \& Aslin, R. N. (2000). Spoken word recognition and lexical representation in very young children. Cognition, 76, 147-166.

Tan, S. H., \& Schafer, G. (2005). Toddlers' novel word learning: Effects of phonological representation, vocabulary size and parents' ostensive behaviour. First Language, 25(2), 131-155.

Thomas, D. G., Campos, J. J., Shucard, D. W., Ramsay, D. S., \& Shucard, J. (1981). Semantic comprehension in infancy: a signal detection analysis. Child Development, 52(3), 798-903.

Tincoff, R., \& Jusczyk, P. W. (1999). Some beginnings of word comprehension in 6-month-olds. Psychological Science, 10(2), 172-175.

Tummeltshammer, K. S., Wu, R., \& Kirkham, N. Z. (2013). 8-month-olds Know Which Face is Reliable. Paper presented at the Proceedings of the Annual Meeting of the Cognitive Science Society.

van Heugten, M., \& Johnson, E. K. (2014). Learning to contend with accents 
in infancy: Benefits of brief speaker exposure. Journal of Experimental Psychology: General, 143(1), 340.

Walker-Andrews, A. S., Haviland, J. M., Huffman, L., \& Toci, L. (1994).

Brief report: Preferential looking in intermodal perception by children with autism. Journal of Autism and Developmental Disorders, 24(1), 99.

Werker, J. F., Fennell, C. T., Corcoran, K. M., \& Stager, C. L. (2002). Infant's ability to learn phonetically similar words: Effects of age and vocabulary size. Infancy, 3(1), 1-30. 